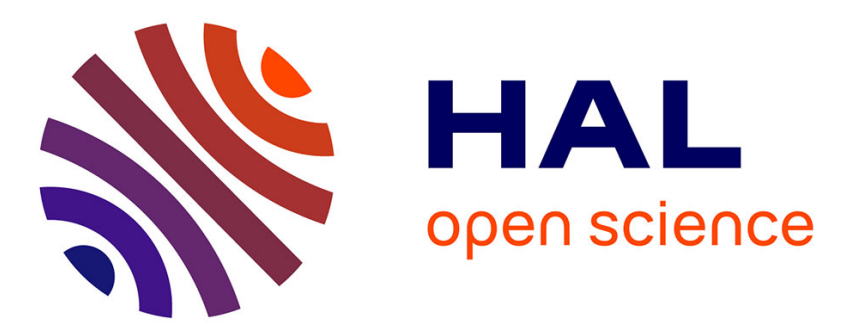

\title{
La production discursive des normes: centralité sociolinguistique et multipolarisation des espaces de référence
}

Thierry Bulot

\section{- To cite this version:}

Thierry Bulot. La production discursive des normes: centralité sociolinguistique et multipolarisation des espaces de référence. Journal of French Language Studies, 2006, The dynamics of levelling and diversity in French/Dynamiques de 'standardisations partielles' en français, 16 (3), pp.305-333. halshs-01090764

\section{HAL Id: halshs-01090764 \\ https://shs.hal.science/halshs-01090764}

Submitted on 10 Dec 2014

HAL is a multi-disciplinary open access archive for the deposit and dissemination of scientific research documents, whether they are published or not. The documents may come from teaching and research institutions in France or abroad, or from public or private research centers.
L'archive ouverte pluridisciplinaire HAL, est destinée au dépôt et à la diffusion de documents scientifiques de niveau recherche, publiés ou non, émanant des établissements d'enseignement et de recherche français ou étrangers, des laboratoires publics ou privés. 


\section{LA PRODUCTION DISCURSIVE DES NORMES : CENTRALITE SOCIOLINGUISTIQUE ET \\ MULTIPOLARISATION DES ESPACES DE REFERENCE}

Thierry Bulot

Université Rennes 2 /ERELLIF-CREDILIF EA 3207

\section{INTRODUCTION : DU NIVELLEMENT ET DE LA CENTRALITÉ}

Les notions de nivellement dialectal ou linguistique et celle de centralité linguistique procèdent, pour le moins, d'une même activité terminologique visant - par la métaphorisation opérée à partir de faits et d'observations spatiaux - à rendre compte de processus sociolinguistiques spécifiques mais a priori aussi proches que lointains... Proches, car impartis aux langues et à leurs variétés, les deux termes - si on en reste à filer la métaphore - font état pour le nivellement d'une sorte d'aplanissement des formes d'un relief langagier contrasté et pour la centralité d'un repérage desdites formes dans une spatialité horizontale dès lors construite. Lointains, car même si le premier terme renvoie, pour la compréhension de son acception (Foulkes et Docherty, 1999: 13), à la mobilité sociale et spatiale ${ }^{1}$, il exclut de l'analyse de la hiérarchisation sociale des variétés et de leur éventuelle mise à distance linguistique, les effets-mêmes de l'épaisseur socio-spatiale sur le processus. C'est ce que tente évidemment de poser le concept de centralité linguistique qui spécifie «...l'attitude qui consiste à placer en un lieu (...) la forme de prestige sans pour autant que soit attestée la dite forme.» (Bulot, 2001 : 37), et permet de saisir, dans les rapports dialectiques entre discours sur la/les langue(s) et discours sur les espaces et les lieux, autant la discrimination et surtout la ségrégation socio-spatiale mise en mots par les discours épilinguistiques que, pour permettre l'identification des locuteurs à un espace partageable, la nécessité communautaire à produire de l'unique et de l'identitaire, c'est-à-dire un espace et une norme de référence.

Précisément, l'espace qui, ici, nous intéresse est la ville. Pourquoi ? parce que, dans les rapports à la normalisation non verbale tant des attitudes que des comportements, elle "...représente un des moyens utilisés par un organisme social pour contrôler et maintenir sa structure» (Laborit 1971 : 27) ; dans le cadre de cet article la ville nous intéresse parce qu'elle est l'espace privilégié du nivellement linguistique. Effectivement, si cet espace méta-social a nécessairement pour objet de contrôler la dynamique des langues, voire d'en organiser l'émergence (Lodge, 1999), on doit raisonnablement pouvoir affirmer qu'il est aussi celui de la production des normes (Andersen, 1998 ; Dauzat, 1906) ou, à tout le moins, celui des discours sur la qualité urbaine de la norme du français.

Pour ce faire, en référence à des enquêtes de terrain, l'article propose de questionner la production discursive des normes (les discours tenus sur les façons de parler d'autrui ou de soi-même) sous l'angle de la multipolarisation des espaces de référence. Nous allons ainsi dans un premier temps rendre compte d'enquêtes épilinguistiques effectuées dans trois configurations urbaines distinctes : 1 . le discours sur l'accent de Rouen, forme linguistique identitaire et en même temps stigmatisée, 2. le discours sur le cauchois d'Yvetot (le cauchois est une des variétés du normand), et 3. le discours sur les langues à Rennes (entre autres, autour de la langue française et des langues bretonnes).

${ }^{1}$ Le nivellement linguistique semble être un des faits issus de la mobilité des personnes vers la ville et se situer dans le paradigme du brassage des langues et des parlures. 
Dans un second temps, après avoir rappelé les propositions théoriques concernant la spatialité en sociolinguistique urbaine, l'article présente une modélisation inédite de l'espace urbanisé pour tenter de mieux saisir les spécificités des dynamiques discursives impliquées par la culture urbaine. Il commente, en regard avec le concept de nivellement, les conceptualisations qui sont à la base d'une telle approche, et notamment la centralité linguistique comme processus producteur de normes et son corollaire quasi dichotomique, le confinement linguistique, comme processus vecteur de contre-norme. Pour ce qui est des terrains que nous avons étudiés, il est en effet constant de constater que tout locuteur déclaré d'une communauté sociolinguistique est en mesure d'inscrire son discours identitaire selon deux modalités corrélées : la territorialisation linguistique et la hiérarchisation des espaces.

\section{LA MULTIPOLARISATION DES ESPACES}

\section{Cadrage des études ou du marquage langagier des espaces}

Les situations dont nous allons rendre compte ${ }^{2}$ ont en commun d'être dans une zone francophone spécifique : d'une part elles sont situées dans la partie ouest de la zone d'oil de la France et d'autre part elle possèdent chacune une identité linguistique et culturelle suffisamment affirmée pour donner lieu à des discours en relevant. Par ailleurs, leur proximité géographique permet de mieux concevoir le continuum existant entre les différentes formes linguistiques; il s'agit donc de la Normandie (en fait la partie nord, dite la Haute-Normandie et particulièrement le Pays de Caux) et de la Bretagne (en fait la Bretagne gallèse, dite la HauteBretagne $)^{3}$. Existe dans chacune de ces zones un continuum finalement peu spécifique allant de formes perçues comme locales (respectivement le cauchois et le gallo) à une forme de français perçue comme standard et relevant davantage d'un français régional normé, dit plus urbain que rural, avec lequel il est confondu la plupart du temps. Dans les deux situations francophones, les formes les plus proches des langues locales ont à la fois un statut de langue identitaire (et sont en cela des dialectes-langues au sens strict du terme) et à la fois un statut de formes catégorielles (et sont de ce point de vue des sociolectes). Tantôt dialectes sociaux, tantôt sociolectes dialectaux, les formes marquées comme les plus proches des langues d'oiil ${ }^{4}$ locales (cauchois et gallo) donnent pour le moins lieu à des discours, et c'est - de notre point de vue - ce qui permet de les approcher ensemble sans pour autant les confondre ${ }^{5}$, car nous verrons que leurs spécificités sociolinguistiques ne sont pas des moindres. Dans le cas où les locuteurs n'ont ni la conscience ni le discours identitaire sur la langue locale, cas qui constitue la majeure partie des locuteurs, les pratiques langagières et linguistiques sont considérées (en auto ou en hétéro-désignation) comme étant du français plus ou moins normé.

Cela étant, nous allons montrer que les faits relevant de l'urbanité langagière ont comme action, parce qu'ils renvoient à une matrice discursive renvoyant toujours à une altérité

2 Pour ne pas alourdir le propos dans les commentaires qui suivent, nous ne revenons pas (sauf si c'est absolument nécessaire pour la compréhension), sur les échantillonnages, les protocoles,... qui ont amené aux résultats que nous allons relater. Cela a été fait dans d'autres publications auxquelles nous renverrons au fur et à mesure du texte. Rappelons seulement que les enquêtes procèdent soit de questionnaires, soit d'enquêtes par entretien semi-directifs et qu'elles portent sur les attitudes langagières (même si les aspects linguistiques sont présents). Autrement dit, les résultats que nous présentons relèvent d'une méthodologie globale proche de l'analyse du Discours (Bulot et Veschambre, 2006).

3 Trois sites urbains vont être abordés: Rouen (préfecture la Seine-Maritime et de la Haute-Normandie), Yvetot (petite ville du Pays de Caux considérée par les Cauchois comme leur capitale linguistique) et Rennes (préfecture d'Ille-et-Vilaine et de la Bretagne).

${ }^{4}$ Le terme «marquées » signifie que lesdites formes sont celles construites dans les pratiques et discours des locuteurs comme du cauchois ou du gallo. On est là dans la prototypie et non dans une différenciation structurelle absolue.

${ }^{5}$ Notons que ces formes relèvent, d'un autre point de vue, de la catégorie des langues collatérales (Eloy, 2004).

${ }^{6}$ Sans le terme espace qui le précède, ce terme est ici à prendre au sens commun de « qui se rapporte à la ville». 
linguistique relative (Bulot, 2003a), non seulement de produire ou reproduire des espaces de références spécifiques d'une variété normée locale qui devient la figure métonymique de la norme, voire de la langue commune, mais encore de hiérarchiser - de manière différenciée les variétés en fonction des espaces normatifs ainsi constitués.

\section{Le français à Rouen : une quadruple centralité}

Pour définir succinctement mais sûrement la ville de Rouen, il suffit de mentionner que c'est la ville de France qui possède - toute comparaison faite - le taux de ségrégation socio-spatiale le plus élevé ; d'un point de vue discursif, cela renvoie à des propos affirmant que la rive droite de la ville possède tous les atouts et valorise ceux qui l'habitent, tandis que la rive gauche est largement dépréciée et stigmatise ses habitants. Existent par ailleurs des discours identitaires sur l'existence d'un accent de Rouen pourtant sis sur la rive gauche ${ }^{7}$, lieu de tous les défauts de la ville.

Les enquêtes « rouennaises » ont connu trois temps ${ }^{8}$ : le premier $(1994 / 1998)$ a décrit les fractures urbaines à Rouen en regard avec quatre autres villes européennes (Berlin, Venise, Athènes et Mons) ; ce premier moment a eu comme objectif d'élaborer une grille conceptuelle et analytique. Le deuxième temps (1996/1999) a porté sur l'émergence discursive des catégories de l'espace urbain à Rouen et a été mis en place auprès d'une population rouennaise non migrante (en fait dont l'identité ne se décline pas via la migration). Le troisième temps (1998/2002) a confronté les données recueillies précédemment avec celles issues d'un échantillonnage de population étendu aux populations migrantes et/ou qui se perçoivent comme originaires d'une migration récente'. Dans les trois cas, il s'est agi de recueillir les attitudes langagières de locuteurs rouennais selon une méthode aménagée du locuteur masqué. Un jeu de bandes ${ }^{10}$ ad hoc a été soumis à des locuteurs témoins qui avaient pour tâche d'évaluer (par le biais d'échelles évaluatives) ou de dénommer (par élicitation d'items qualifiants) et les variétés et les catégories qui leur étaient soumises ou non.

Les résultats obtenus auprès des Rouennais non-migrants (résultats qui ne se limitent pas à cela bien entendu) sont à la fois attendus et étonnants. Attendus, car les discours tenus sur l'accent de Rouen et, partant, le français standard, ne sont pas différents des stéréotypes: à Rouen, on parle français - et plus tôt bien - et (sans doute 'mais') l'accent de Rouen (le discours sur l'accent de Rouen) permet de différencier nettement une rive droite de la ville où l'on parle bien le français, d'une rive gauche où on le parle mal. Attendus certes, mais seulement si on considère que les stéréotypes renvoient à la dominance socio-langagière : il n'est en effet pas étonnant que les locuteurs de la rive droite de la ville (Figure 1) s'auto-attribuent le français normé (FRN) en le localisant non seulement sur la rive droite mais encore au centre-ville droit, à Rouen et en ville. L'espace de référence valorisant est a priori celui-là. Le placement de la variété stigmatisée (FRS, l'accent de Rouen) est conforme eu égard aux stéréotypes la concernant : de la rive gauche, rouennais, urbaine et en centre-ville gauche. Ils repoussent sur l'autre rive toutes les formes qui ne sont pas perçues comme normées.

\footnotetext{
${ }^{7}$ C'est une configuration récente. Mais le terme rive gauche est un choronyme socionymique (Bulot, 2004a et 2004b) permettant de discriminer une population ouvrière. Le quartier anciennement dit «Les Sapins » est dit de la rive gauche dans nos enquêtes parce qu'il est un quartier dit sensible, alors qu'il est géographiquement sur la rive droite de la ville.

${ }^{8}$ Nous allons commenter les résultats du deuxième et troisième temps.

${ }_{9}^{9}$ Pour plus de précisions, consulter le lien : http://www.sociolinguistique-urbaine.com/op12.htm

${ }^{10}$ Dans les figures 1 et 2 les bandes FRN (français rouennais normé), FRC (français rouennais courant), FRS (français rouennais stigmatisé), FIA (français rouennais africain i.e. d'Afrique de l'Ouest) et FIM (français rouennais maghrébin) concernent le temps 2 de nos enquêtes. Dans les figures 5 et 6 , on retrouve la même méthode avec en partie les mêmes bandes (seulement FRS, FIA et FIM) avec deux nouvelles : FIT (français rouennais turc), FIO (français rouennais asiatique i.e. originaire d'Extrême-Orient). RD et RG mis en exposant signifient respectivement les locuteurs de la rive droite ou les locuteurs de la rive gauche.
} 


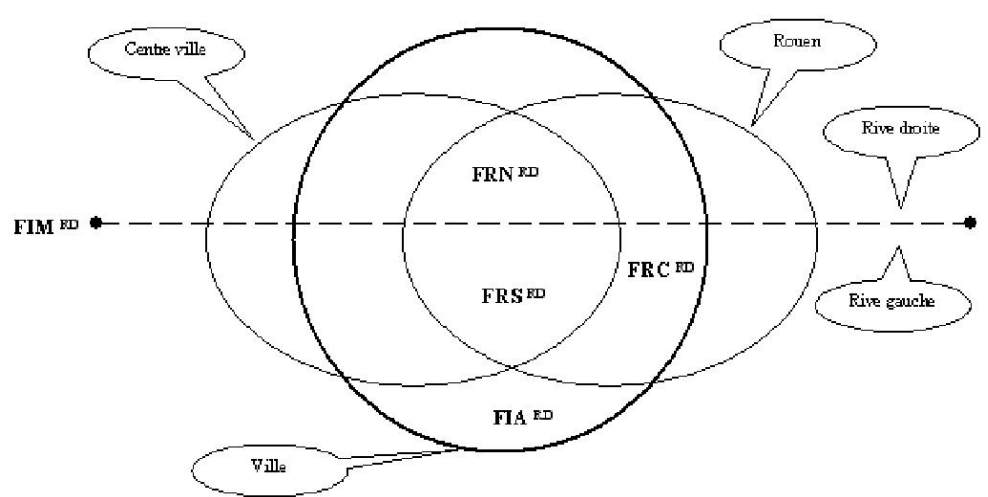

Figure 1. L'engagement normatif positif (rive droite)

Étonnants dès lors que l'on considère les réponses des locuteurs de la rive gauche (Figure 2). Ils assument de fait pour eux-mêmes, pour leur espace de référence, la stigmatisation : aucune des variétés régionales non marquées par l'immigration n'est localisée sur la rive gauche. C'est évidemment sur la rive droite que l'on parle mieux, ou dit autrement, ça n'est pas sur la rive gauche que l'on parle bien. Mes ces premiers constats appellent des commentaires plus précis. D'abord, la variété rouennaise stigmatisée (FRS) reste rouennaise et urbaine mais n'est pas assumée comme étant effectivement de la rive gauche de la ville; le stigmate est ainsi en partie assumé et en partie inversé. Plus encore, la forme normée de français (FRN), même si elle demeure de la rive droite pour signaler sa proximité du standard, n'est plus du centre ville de Rouen, n'est plus rouennaise du tout. L'espace de référence des locuteurs de la rive gauche n'est pas rouennais mais celui des centres-villes valorisés.

On constate ainsi deux premiers niveaux de centralité sociolinguistique: selon que les locuteurs se définissent, s'identifient comme étant d'une rive ou d'une autre de la ville, d'une portion spécifique de l'espace commun, ils n'ont pas la même représentation de l'espace tant social que - surtout - celui impartis aux variétés de langue. L'hétérogénéité constitutive de l'espace commun est de la sorte mise à jour: Rouen est en partie le discours tenu sur les structures socio-spatiale et l'accent de Rouen, et en partie les discours relevant de contrenormes tant langagières que spatiales. C'est à la fois la même ville et à la fois une ville différente.

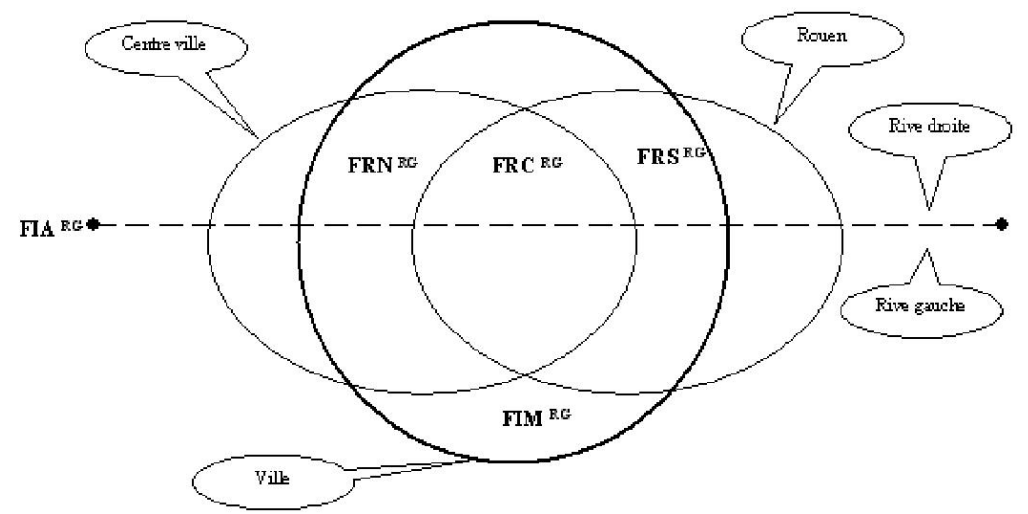

Figure 2. L'engagement normatif positif (rive gauche)

Les résultats obtenus auprès des Rouennais migrants permettent de préciser plus encore ces constats. Les localisations des variétés FIA et FIM du temps précédent (Figures 3 et 4) ont nettement donné à voir une externalisation: les formes perçues comme marquées par les migrations ne peuvent être identifiées comme rouennaises (car peu patrimoniales à ce jour), comme de la rive droite (car stigmatisant l'exclusion, la relégation, ou comme du centre ville (car situées à l'opposé des pratiques normées). L'enquête faite auprès de migrants (essentiellement des personnes issues du Maghreb ou d'Afrique de l'Ouest) fait apparaittre 
l'efficience, comme une constante dans l'identité rouennaise, de la ségrégation socio-spatiale posant une rive droite valorisante et une rive gauche stigmatisante. C'est en effet l'habitat déclaré des locuteurs (et non leur origine ethnique, leur francophonie originelle, leur sexe, leur niveau d'étude...) qui va déterminer leurs évaluations ${ }^{11}$.

Les migrants de la rive droite (Figure 3) identifient nettement la forme stigmatisé (FRS) en accord presque parfait avec le stéréotype : elle est de la rive gauche, du centre-ville gauche et rouennaise mais elle n'est pas envisagée comme urbaine sans doute dans la mesure où la forme n'est pas soit perçue comme suffisamment normée soit considérée comme quasi rurale (ce qui revient presque au même dans les représentations dominantes). Sur la même rive est identifiée la forme urbaine FIA. La configuration est donc la suivante : sur la rive gauche, co-existent des formes non normées de français, marquées ou non par l'immigration. La rive droite, pour sa part, est spécifique de deux formes : la première (FIT), posée comme rouennaise, appelle un commentaire d'importance ; en effet, elle a été reconnue comme une forme «maghrébine » (et non turque), et dans ses attributs (le métier supposé, le niveau d'études,...) elle est fort proche de la forme FRS telle qu'elle avait été évaluée dans le temps par les locuteurs de la rive droite. La seconde (FIM) prend fonction et place de la forme FRN ${ }^{\mathrm{RD}}$ : elle est décrite comme non marquée par un accent, comme une forme spécifique des catégories socioprofessionnelles les plus élevées (cadre, avocats, médecins, enseignants...) ; autrement dit, alors qu'elle supportait fortement des attributs négatifs dans le temps 2 des enquêtes, cette forme devient la norme de référence pour les personnes interrogées: elle est effectivement, en auto-attribution, du centre-ville, sur la rive droite, urbaine et rouennaise.

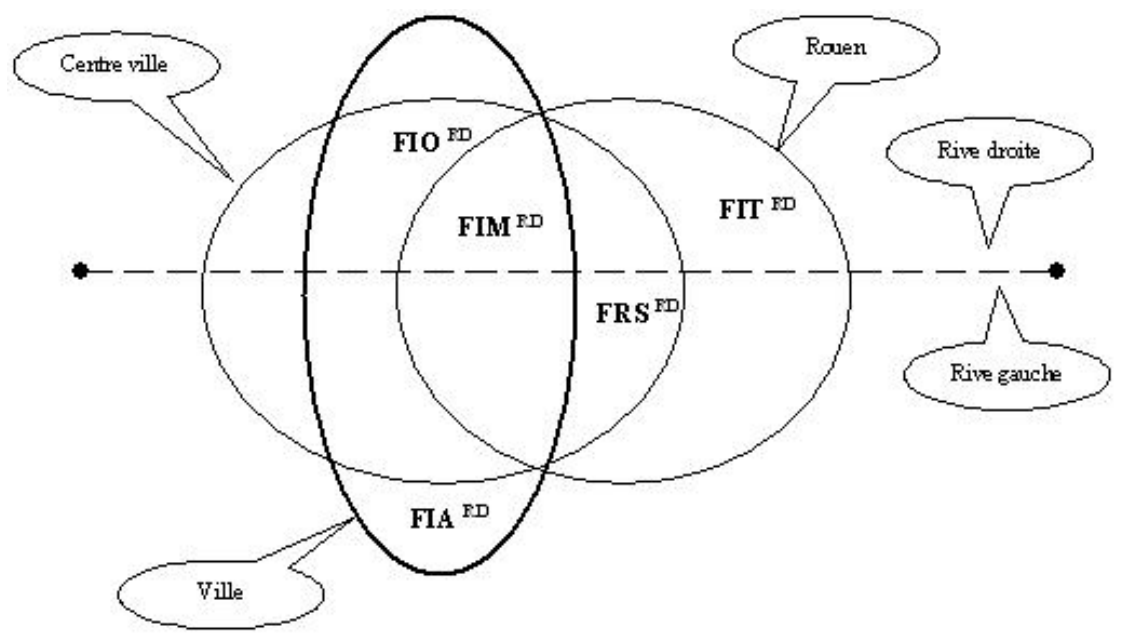

\section{Figure 3. L'engagement normatif positif (les migrants de la rive droite)}

La comparaison de ces réponses avec celles des migrants de la rive gauche (Figure 4) produit un effet de cohérence certain :

a) la forme de référence reste la même et fait montre d'une identification nettement différenciée d'avec les évaluations des non migrants; FIM est, d'une certaine manière, la forme identitaire commune et construite comme la variété normée rouennaise, et sans doute francophone (quand bien même elle est stigmatisée par ailleurs, ce qui est un des aspects du confinement linguistique, $c f$. infra).

\footnotetext{
${ }^{11}$ On doit noter à part le traitement de la forme FIO, qui sur les deux rives (Figures 5 et 6) va caractériser le centre-ville de la rive droite; il convient d'ajouter que l'activité professionnelle systématiquement déclinée est « restaurateur » et ses dérivés. Il va de soi qu'une telle typification (i.e. sans plus de nuance) cache une réalité plus complexe; elle signifie au moins que les Rouennais migrants considèrent cette population autrement qu'euxmêmes.
} 
b) Hors la forme FIO (qui demeure atypique mais caractéristique, cf. note 25), les autres formes marquées ou non par l'immigration (qui sont mêmement décrites quant aux attributs sociaux) sont localisées sur la rive gauche et rouennaises. Là où les nonmigrants récusaient pour leur espace les formes non-normées, les migrants les acceptent et se les attribuent.

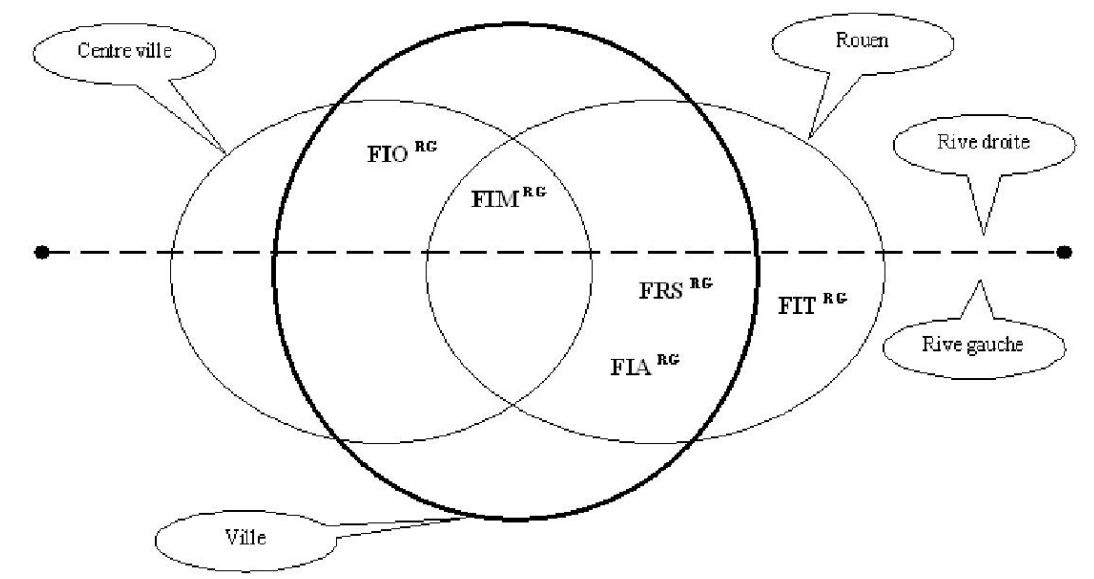

Figure 4. L'engagement normatif positif (les migrants de la rive gauche)

On constate ainsi un second niveau de centralité linguistique : non seulement les nonmigrants déclinent différemment leur espace urbanisé sur une opposition rive à rive mais encore les migrants territorialisent ce même espace sur la même opposition en reconnaissant tendanciellement comme rouennaises les formes considérées par les premiers comme les moins locales. Plus encore, leur forme de référence, si elle est identifiée sur une même catégorisation socio-spatiale, si elle est décrite comme la norme valorisante, se trouve être formellement et attitudinalement deux variétés distantes mais, en discours, représentées comme étant une seule et même variété.

\section{Le continuum français / cauchois : une centralité urbaine et post-diglossique}

Le cauchois est le glossonyme de la variété de normand du Nord parlé approximativement sur le département de Seine Maritime (France) qui comprend pour sa part le Pays de Caux proprement dit flanqué au Nord du Petit Caux, au Nord-est du Pays de Bray et au sud de la Vallée de Seine. Ses limites nord sont délimitées par la côte maritime (La Manche) et au sud par les falaises crayeuses de la vallée de la Seine (Figure 5). Les limites Est sont moins nettes au moins du point de vue sociolinguistique, non seulement en raison d'un continuum perceptible et presque nécessaire dans les formes perçues comme locales (ne serait-ce que parce que le brayon - le parler du pays de Bray - est aussi proche linguistiquement du picard que du cauchois) mais encore parce que les discours identitaires sont essentiellement focalisés sur deux espaces linguistiques aux contours et limites institutionnelles et culturelles peu nettes sur leur point de rencontre : le Pays de Caux et le Pays de Bray.

Les enquêtes cauchoises ont connu deux temps importants :

1. un ensemble d'entretiens semi-directifs effectués auprès de Cauchois pour tenter d'approcher les représentations sociolinguistiques des pratiques socio-langagières locales (1998-1999) complété d'une enquête (en observation participante) effectuée en milieu scolaire ${ }^{12}$ pour essayer d'évaluer les compétences de jeunes enfants en zone suburbaine (2000), d'une part, et, d'autre part

12 Voir le compte rendu de cette recherche dans Bulot, T. et Jannot, L. (2001). Hétérogénéité linguistique en milieu scolaire. Les pratiques linguistiques régionales en Pays de Caux. Moderne Sprachen ,45/1 38-52. 
2. un questionnaire dispensé auprès de lecteurs d'un hebdomadaire cauchois à fort tirage (et qui donne à lire une rubrique en cauchois ${ }^{13}$ ) (2003) et, auprès de lycéens, collégiens et étudiants du Pays de Caux d'autre part (2004).

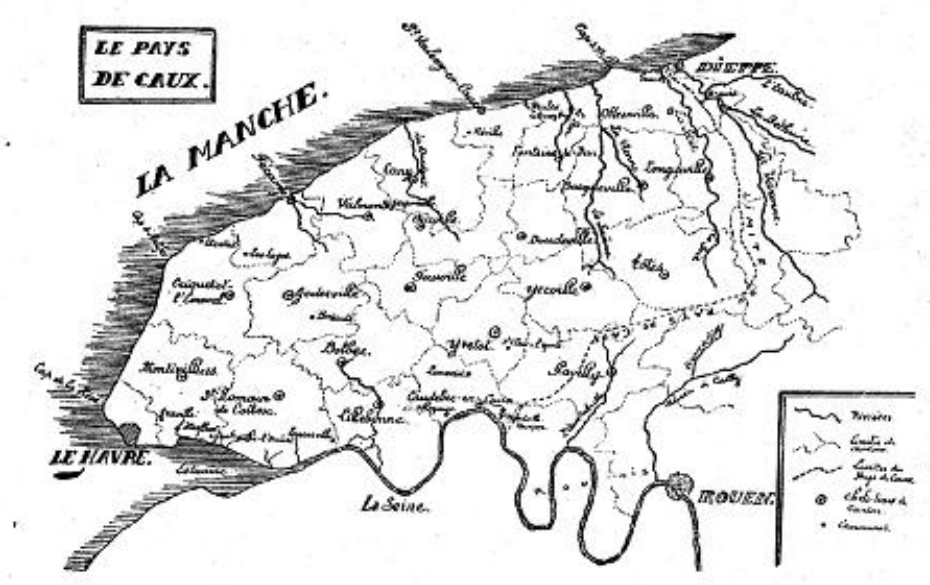

Figure 5 : Le Pays de Caux (Mensire, $1984: 4$ )

Cela posé, les stéréotypes socio-langagiers concernant le cauchois rendent compte de la déconsidération sociale d'une forme réservée aux ruraux - donc à un espace morphologiquement et socialement rural - et / ou aux plus âgés - donc à un espace qui plus est générationnel - et / ou les moins cultivés - donc à un espace social dévalorisé. Synthétiquement, le discours social fait du cauchois une forme parlée et non-écrite par des vieux ruraux peu cultivés ce qui explique - compte tenu, en outre, de la rurbanisation ${ }^{14}$ des campagnes - sa nécessaire et prochaine disparition du paysage linguistique français. Nous ne revenons pas à présent sur la totalité voire l'inanité de certains de ces traits ${ }^{15}$, ni ne souhaitons argumenter autour d'une situation paradoxale affirmant au moins depuis le début de $19^{\text {ième }}$ siècle la disparition, sur le territoire de l'État français, des formes romanes autres que le français ${ }^{16}$. En revanche, nous souhaitons montrer comment le cauchois - c'est-à-dire ses locuteurs - construit sa légitimité autour de la centralité sociolinguistique.

Que parle-t-on en Pays de Caux ? Une récente enquête sociolinguistique ${ }^{17}$ (Bulot, 2005b) effectuée auprès de 382 personnes fait état d'une réponse massive $(83 \%)$ indiquant le français comme la langue de la région en parallèle avec le cauchois (67,3\%). S'il est peu étonnant que le français arrive ainsi en premier rang des réponses, cela l'est davantage pour le cauchois. Eu égard à des situations antérieures (Marcellesi et Lozay, 1983), il semble manifeste que la configuration sociolinguistique a changé ces dernières décennies, non pas tant en termes de pratiques, mais surtout en termes de discours sur les pratiques. Nous ne revenons pas sur le détail de ces conclusions d'enquête, mais on assiste peut-être à une étape spécifique du processus de nivellement linguistique ; en effet, l'un des effets de ce processus, qui est en partie un pan de la mise en diglossie des variétés d'oil, est de faire concevoir le cauchois

\footnotetext{
${ }^{13}$ Le Courrier Cauchois (Seine-Maritime / Yvetot).

${ }^{14}$ Le terme renvoie ici à l'émergence de zones rurales habitées par des urbains qui y imposent leur comportements et normes sociales.

${ }^{15}$ Voir Bulot, T. et Courard, S. (2001). Patrimoine et langue : modernité dialectale et identité linguistique (Pays de Caux). Europa Ethnica, 58/1-2, 58-63; et Bulot, T. (2004). Pratiques langagières en Pays de Caux: faits de dominance et glottopolitique. Moderne Sprachen ,48/2, 59-74.

${ }^{16}$ Bulot, T. (1989). L'enquête de Coquebert de Montbret et la glottopolitique de l'Empire français. Romanischen Philologie, 2-89, 287-292.

${ }^{17}$ Qui correspond au second temps d'enquête évoqué ci-dessus.
} 
comme une variété - basse en général - de français. Or le fait de pouvoir déclarer le cauchois comme la langue de la région ${ }^{18}$ procède d'une conception de la forme comme entité autonome, distante sociolinguistiquement du français, sans pour autant être d'ailleurs en conflit avec lui. Les résultats concernant la compétence déclarée corrélée à la compétence effective montrent de surcroît une étonnante mais logique cohérence interne des attitudes des Cauchois : plus ils se déclarent compétents en cauchois, plus ils le sont et plus ils affirment son existence.

Pour ce qui est du présent article, il faut noter que ce que montrent nos enquêtes est que le cauchois est aussi parlé en ville et constitue - c'est une donnée qui paraît inédite pour la part valorisante qu'il s'octroie - l'une des formes de référence à côté du français régional urbain parce qu'il constitue une des modalités discursives de l'identité ethno-sociolinguistique (Bulot, 2003b). Non seulement existent des formes urbaines de cauchois (le cauchois de Rouen, le cauchois de Dieppe, ....) mais encore est posé - dans la hiérarchisation des lieux urbains qui fonde le territoire de la forme cauchoise - un stéréotype récurrent : la ville d'Y vetot est la capitale du Pays de Caux, tant du point de vue linguistique que symbolique. Les discours tenus donnent à voir un centre reconnu comme le lieu d'une norme linguistique locale valorisante et statutairement légitime au sein d'un espace francophone. Bien sûr, il ne s'agit pas de dire que tous les Yvetotais parlent (et mieux) et ou comprennent (et mieux) le cauchois mais que la grande majorité de locuteurs qui se déclarent cauchois (par la simple et première attache géographique) reconnaissent à ce lieu urbanisé et sa périphérie la capacité à être le lieu d'occurrence de la forme linguistique de référence, d'une forme en quelque sorte de cauchois central, normé, incontestable... qui peut devenir la norme de référence du cauchois.

La notion même de continum impose de penser pour les discours épilinguistique une polarisation entre forme basilectale et acrolectale ${ }^{19}$; pour le cas du cauchois et de ses rapports au français, il est manifeste que perdure une transmission, une identité, des pratiques décrites comme spécifique en désaccord presque total avec le discours alarmiste de nombre de militants linguistiques et avec le discours monolingue dominant; ainsi les descriptions sociolinguistiques des années passées ${ }^{20}$ posent la configuration suivant : en Pays de Caux, le français est la forme haute et le cauchois la forme basse locale. La situation actuelle semble différente dans la mesure où se construit une conscience linguistique qui semble sinon sortir absolument de la diglossie du moins faire état d'un processus de reconnaissance-naissance d'une langue pour des pratiques régionales identifiées par les chercheurs comme révélatrices du continuum local et pratiquées par les locuteurs comme leur langue.

Concrètement, les locuteurs déclarés du cauchois territorialisent l'espace commun, de fait se l'approprient en investissant les espaces urbanisés ${ }^{21}$ et hiérarchisent ce même espace non plus en regard avec une territorialité ruralo-patrimonio-folklorique mais avec pour traits définitoires un ensemble hiérarchisé de villes fonctionnant en réseau et donnant à percevoir et à pouvoir dire une identité. La centralité sociolinguistique est un fait urbain et urbanisé en Pays de Caux et, même si celle-ci se construit dans une configuration en partie diglossique dans la mesure où la capitale n'est pas la capitale régionale de fait - c'est pour faire cas d'une identité sociolinguistique positive, valorisante et de plus en plus assumée.

\footnotetext{
${ }^{18}$ D'autant que plus de la moitié de l'échantillon a moins de 20 ans.

${ }^{19}$ Les formes intermédiaires (mésolectales) étant attribuées à tel ou tel pôle suivant, entre autres, les stratégies identitaires en œuvre.

${ }^{20}$ En fait celles qui se situent avant le débat sur Charte européenne des langues régionales ou minoritaires.

${ }^{21}$ On ne peut plus concevoir que le jeune Cauchois est un rural sans contact avec les formes de culture urbaine...
} 


\section{Le bilinguisme à Rennes ${ }^{22}$ : une centralité doublement minorante}

La ville de Rennes constitue un autre cas spécifique pour l'approche de l'urbanité langagière. D'abord 1) parce que contrairement à Rouen marquée par la ségrégation socio-spatiale, la ville est, pour sa part, une championne déclarée (et par ailleurs attestée par les données officielles) de la mixité sociale (Guy et Givord, 2004) ; 2) parce que son statut de ville universitaire a pour corollaire que la proportion des 15-29 ans y atteint les 34\% contre 19,9\% à l'échelon national ; et 3) parce que, même si le nombre d'étrangers y est moindre eu égard à la situation française (4\% contre 5,6\%), il convient de noter que la ville accueille à elle-seule $26 \%$ de l'ensemble des étrangers de la région Bretagne et plus encore plus de $66 \%$ des étrangers du département d'Ille-et-Vilaine. Des langues autres que le français y sont ainsi diversement attestées. Ensuite, elle se situe dans une région à forte identité linguistique (la Bretagne et spécifiquement la zone gallèse de la Bretagne) qui implique, pour sa part, sinon des pratiques du moins des discours sur la présence d'une langue régionale commune - le breton, langue celtique -et surtout la coprésence d'une langue collatérale à base romane, le gallo, souvent identifiée par ses locuteurs comme une forme courante voire dévalorisée de français ${ }^{23}$.

Nous ne revenons pas maintenant sur le détail de ces premières enquêtes ${ }^{24}$ (voir Bulot, 2005a et 2006) autant pour les protocoles retenus que pour les analyses effectuées dans la mesure où ils renvoient à des données concernant essentiellement l'affichage des langues dans l'espace rennais. Retenons néanmoins que ces protocoles intègrent les cartes comme traces d'un discours multiforme sur l'espace. La carte que nous utilisons ci-après (Figure 6) est ainsi produite et utilisée par des agences immobilières rennaises. Ses divisions renvoient doublement à la toponymie et au coût du foncier. Nous souhaitons ici faire brièvement état des discours sur la centralité sociolinguistique rennaise pour ce qu'ils rendent compte d'une multipolarisation de l'espace commun corrélé aux langues identifiées.

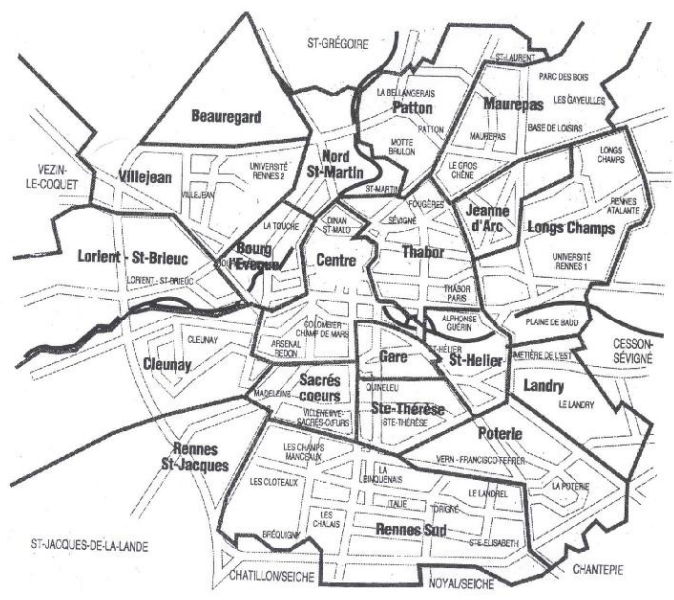

Figure 6. La carte rennaise

22 Les recherches sur la ville de Rennes et son agglomération en sont à leur début : un premier protocole a été élaboré et mis en place dans le cadre de l'Action Concertée Incitative (2004-2005) Espaces et Territoires sur le projet «Mémoire et mise en mots de l'habitat dit populaire». Un programme de recherche spécifique (Multilinguisme et ségrégation : altérité linguistique, mémoire sociolinguistique et politique de la ville) à notre laboratoire a été initié en 2006 dans le cadre d'une collaboration avec les collectivités locales (Rennes Métropole).

${ }^{23}$ Voir les travaux de Francis Manzano (1997) et de Nathalie Trehel et Philippe Blanchet (2003) pour un état de la question.

${ }^{24}$ Voir Bulot, T. (2005). Discours épilinguistique et discours topologique : une approche des rapports entre signalétique et confinement linguistique en sociolinguistique urbaine. Revue de l'Université de Moncton, Vol 36/1, 219-255. Et Bulot, T. (2006). Discrimination et processus discursifs de fragmentation des espaces urbains (Signalétique et bilinguisme). Dans: Mots, traces et marques (Dimensions spatiale et linguistique de la mémoire urbaine). Paris : L'Harmattan (Collection Espaces Discursifs), pp. 97-124. 
Lorsque les locuteurs que nous avons interrogés ont eu à dénommer les lieux de la norme ("Où parle-t-on le mieux le français à Rennes?»), ils ont, de fait, mis en mots un espace de référence, une centralité sociolinguistique en partie conforme aux stéréotypes impartis aux villes du Nord-Ouest de la France et qu'on a déjà trouvés à Rouen : la norme est au centreville ; en partie seulement car c'est un autre quartier, proche du Centre mais nettement distinct, qui possède pleinement les attributs positifs de la référence socio-spatiale : le Thabor.

Cette distinction est d'importance car elle signale une disjonction entre une centralité urbaine correspondant à la mise à disposition d'un espace collectif (le centre-ville en grande partie historique donc touristique), et une centralité sociolinguistique posant une portion de l'espace rennais comme l'espace de référence absolu pour le français normé. Une telle disjonction introduit une rupture qui semble corroborée par les discours tenus sur le contreespace de référence ("où parle-t-on le plus mal le français à Rennes?») qui exclut du centreville et du Thabor (et plus largement des zones proches du Centre) toutes formes normées de français, les formes régionales romanes (donc gallèses) et les formes de français perçues comme marquées par les migrations récentes ou en cours. Sont ainsi désignés les quartiers Rennes Sud (majoritairement) et Villejean (dans une moindre mesure), avec pour corollaire et justificatif la forte présence perçue d'étrangers. Au bilan, le français de référence est parlé dans deux zones centrales valorisantes et valorisées par l'habitat, le patrimoine architectural, la renommée des équipements culturels,... et les formes stigmatisées dans deux zones périphériques surtout marquées par la présence de migrants et dans une moindre mesure par les classes populaires ainsi que par un habitat récent, collectif.

Cette configuration prend tout son intérêt quand on observe la centralité sociolinguistique des deux langues régionales : le gallo, déjà minoré dans son rapport au français, dans la mesure où il est souvent identifié à la variété basse de la langue nationale, se trouve tendanciellement écarté des espaces de référence de la norme, et relégué dans les quartiers périphériques populaires, ouvriers et pour la plupart non marqués par l'immigration récente (les quartiers Maurepas, Beauregard, Villejean et Lorient Saint Brieux). Cela, non seulement dans son rapport à la langue nationale mais aussi dans son rapport à la langue régionale dominante : en effet, en discours le breton occupe, dans une plus vaste mesure, l'espace tant géographique que social imparti au français de référence (les quartiers ${ }^{25}$ du centre-ville, du Thabor et, en plus, SaintHélier) (Figure 7).

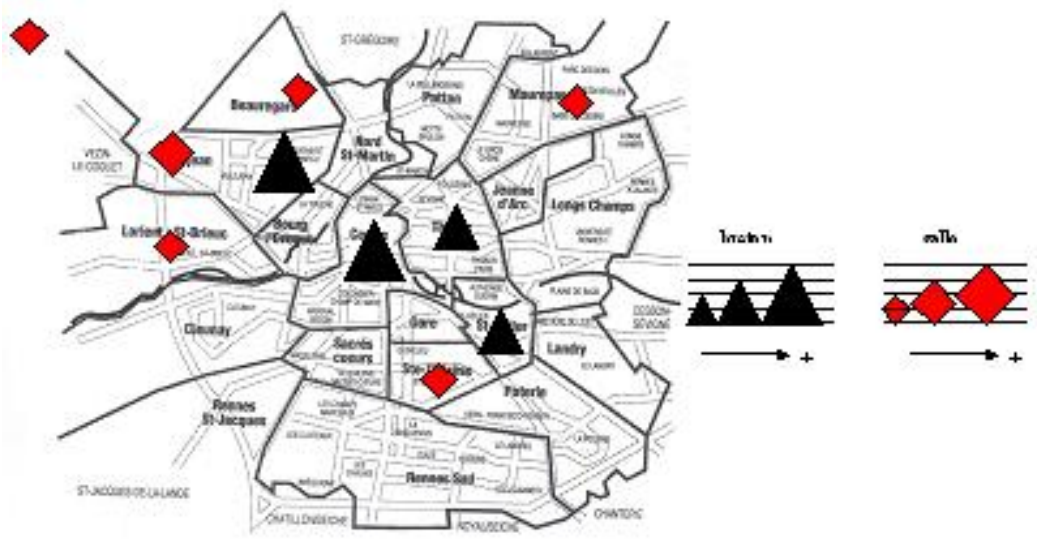

Figure 7 : les espaces de référence du breton et du gallo

${ }^{25}$ Dans cette liste, ne figure pas le quartier Villejean pourtant présent sur la carte ; en effet, il s'agit de nommer l'université de Rennes 2 (Villejean-université et non le quartier lui-même) où sont dispensés régulièrement des cours de breton et dans une moindre mesure de gallo. 
On a donc à Rennes une centralité doublement minorante, celle du français face aux langues régionales, et celle du breton face au gallo, le gallo étant, historiquement, la langue première de la ville. Mais davantage, autour de ce processus partiellement masqué, puisque l'affichage odonymique rennais ${ }^{26}$ ne laisse voir que les contacts entre le français et le breton, c'est la mise en hégémonie du breton comme vecteur de l'épaisseur identitaire de la ville par l'appropriation des espaces urbains dominant socialement. Le breton n'est pas la langue des couches et quartiers populaires (c'est réservé au gallo), mais la langue des citadins urbanisés, cultivés et aisés; en cela, une telle multipolarisation des espaces notifie une violence symbolique vis-à-vis du gallo qui est au moins aussi forte que celle dite subie jadis par le breton vis-à-vis du français.

\section{CONTINUITÉ ET DISCONTINUITÉ DE LA SPATIALITÉ URBAINE :} REPRÉSENTATIONS DISCURSIVES ET MODÉLISATION DES ESPACES DE VILLE.

\section{De quelle sociolinguistique parle-t-on? Positionnement scientifique}

Les concepts exposés dans cet article relèvent d'une voire de la sociolinguistique urbaine qui, nous le verrons plus en détail, ne se limite pas aux seuls espaces dits urbains mais renvoie à la description et à l'analyse sociolinguistique de toute production discursive des espaces sociolangagiers. Ça n'est pas le lieu ici de rappeler la totalité des arguments visant à distinguer une approche sociolinguistique classique de la ville ${ }^{27}$ d'une approche socio-langagière de l'urbanité (Bulot, 2003a) mais rappelons néanmoins que cette distinction est fondée sur une double crise ${ }^{28}$. La première crise est d'ordre épistémique : sans soustraire du champ les travaux sur la diatopie, la sociolinguistique urbaine problématise le spatial comme central dans son approche parce que ce facteur renvoie au chercheur la lecture et l'organisation du social; parce qu'il permet ainsi de discriminer des recherches sociolinguistiques sises en ville (le lieu d'enquête qu'elles sont majoritairement) de recherches en sociolinguistique urbaine qui impliquent de dépasser les clivages inopérants entre ville et campagne. La seconde crise renvoie au social, dans la mesure où la crise des valeurs, la crise des identités, du lien social, magnifiée par la densification de la culture urbaine, interroge également les modèles de ville sur lesquels la discipline travaille. La demande sociale actuelle exprime, au-delà des regards iréniques sur l'espace urbain comme facteur d'unification linguistique et de mobilité sociale ascendante, une réalité que les sciences sociales ne peuvent ignorer : la ville produit du conflit, de l'exclusion, du confinement... Faire de la sociolinguistique urbaine impose au chercheur une éthique de l'engagement dans la mesure où, reprenant ainsi librement les propositions liminaires de Christian Bachmann et Jackie Simonin (1993), son objet, ses tâches sont de tenter de lutter contre l'exclusion des minorités sociales - urbanisées ou non - toutes les fois que le langage est impliqué.

Pratiquement - c'est-à-dire pour tenter de répondre à une telle demande - la sociolinguistique urbaine (Bulot, 2004a) a pour tâches d'étudier, et cela de manière non hiérarchisée et non exclusive, trois axes fondateurs et spécifiques du champ de l'urbanité langagière :

1) la mise en mots de la covariance entre la structure spatiale signifiante et la stratification sociolinguistique d'une part,

\footnotetext{
${ }^{26}$ Nombre de rues du centre-ville de Rennes ont des noms de rue rédigés en français et en breton.

${ }^{27}$ Voir les commentaires critiques de Louis-Jean Calvet (2004) et de Médéric Gasquet-Cyrus (2004).

${ }^{28}$ Bulot T., Bauvois C. (2004). «Présentation générale. La sociolinguistique urbaine : une sociolinguistique de crise? Premières considérations", dans Lieux de ville et identité (perspectives en sociolinguistique urbaine). Paris: L'Harmattan, 7-12.
} 
2) le contexte social des discours (notamment épilinguistiques) de la ville posée comme une matrice discursive, d'autre part,

3) le façonnement réciproque des structures socio-spatiales ${ }^{29}$ sur les comportements linguistiques et langagiers des sujets et des discours (leurs discours) sur l'espace social et la mobilité vécue ou perçue.

\section{Une acception spécifique de l'urbanisation ${ }^{30}$}

La présente contribution s'appuie sur une théorisation visant à considérer d'une part la prégnance de la culture urbaine sur les conduites langagières, et, d'autre part - et plus encore les effets structurants et structurés de la survalorisation discursive ou non de la mobilité spatiale dans les pratiques sociales. Posons provisoirement que cette posture théorique réfère à des espaces urbains ou, plus simplement formulé, à ce qu'il est convenu de nommer la ville ; ainsi, dans notre acception, l'urbanisation sociolinguistique renvoie à un processus complexe ${ }^{31}$ où, dès lors que la morphologie urbaine et la fonction sociale des espaces spécifiques se trouvent disjoints, s'opère une recomposition discursive des espaces autour de la mobilité spatiale qui agit à la fois sur les comportements et les représentations sociolinguistiques (Boyer, 2003).

Dans ce sens du terme, l'espace (social, énonciatif, cognitif...) est envisagé comme un processus et non comme une donnée dans la mesure où les variétés et langues décrites, discursivisées dans un espace multidimensionnel et de facto urbanisé sont l'une des dimensions remarquables de la réalité urbaine, puisque ce qui est dit par des locuteurs qui se rencontrent ou non, se côtoient ou non, sur eux-mêmes ou sur autrui est évidemment à mettre en relation avec les changements et les tensions sociaux en œuvre avec la production discursive des normes.

Dès lors que l'on discute le terme et le processus de nivellement, il importe, en effet, d'interroger ce que la ville permet, à la fois en tant que structure socio-spatiale et à la fois en tant que matrice discursive. Là où les discours relatifs à la dominance font état de la seule présence d'un français construit comme standard ; là où les représentations sociales relatives à l'urbanité font état d'une koinéisation systématique des parlures urbaines; là où, par ailleurs, l'appartenance à un espace commun mais pourtant fragmenté fait sens pour les habitants et usagers d'un tel espace... là où finalement tout locuteur perçoit une hiérarchie des espaces et des langues attribuées, il nous semble essentiel de questionner une urbanité spécifiquement langagière "empreinte du rapport aux langues représentées ou effectivement présentes dans l'espace urbain». Sachant que "le terme même intègre dans le rapport à l'organisation socio-cognitive de l'espace de ville non seulement les pratiques linguistiques elles-mêmes mais aussi les pratiques discursives et notamment les attitudes linguistiques (celles rapportées à la structure de la langue) et langagières (celles liées à l'usage de la structure linguistique)» (Bulot, 2003a : 101-102). La ville est dans cette mesure une matrice discursive, car elle fonde autant qu'elle est fondée par «... des régularités plus ou moins consciemment élicitées, vécues

${ }^{29}$ Guy Di Méo (1990: 17) propose de définir les structures socio-spatiales comme « ...des objets géographiques structurés du type de la localité, du 'pays', de la région et de la nation, plus ou moins nettement délimités dans les représentations individuelles et collectives, mais perçues cependant avec clarté par une large gamme d'acteurs. ».

30 Gabriel Manessy (1990: 23) réfléchissant à la situation des villes plurilingues (essentiellement africaines dans son propos) pose, pour définir l'urbanisation linguistique, deux types de processus à la fois simultanés et, selon lui, antagonistes: "les uns sont liés à la transformation du tissu social qui réduit le domaine d'efficacité des comportements langagiers traditionnels et étend démesurément le champ de la communication interethnique; les autres attestent de la structuration de la masse cosmopolite des usagers du parler urbain en une communauté citadine où la langue devient un moyen d'identification et de catégorisation ». Cette définition, pour pertinente qu'elle soit, pose la ville comme un «déjà-là » et c'est pourquoi nous n'y souscrivons que partiellement et préférons parler d'urbanisation sociolinguistique.

31 Cela renvoie au fait que l'urbanisation sociolinguistique est à la fois multifactorielle et à la fois multivectorielle. 
ou perçues par ses divers acteurs; régularités sans doute autant macro-structurelles (entre autres l'organisation sociale de l'espace) que plus spécifiquement linguistiques et langagières. » (Bulot 2001b : 6).

\section{Une approche nécessairement transdisciplinaire}

Envisager la ville comme un élément central de la problématisation du terrain impose logiquement de questionner les modèles la décrivant et suppose d'inclure effectivement, dans les réflexions théoriques et méthodologiques, des travaux non sociolinguistiques; en tout cas, cela impose une approche nécessairement syncrétique - transdisciplinaire - de l'objet de recherche, qui ne procède ni d'une logique de l'accumulation ni d'une fusion des concepts, mais de la mise en regard de modèles conceptuels et d'analyse où le langagier est fortement présent dans la discipline initiale, cela sans que la focalisation disciplinaire porte sur lui.

Ainsi, pour approcher la complexité inhérente du fait urbain, en plus des travaux relevant de la sociolinguistique générale (Calvet, 1994 ; Labov, 1976, 2002 ; Barbéris, 1994 ; Baggioni, $1994 ; . .$.$) , va-t-on puiser plus spécifiquement et par exemple dans l'écologie urbaine pour$ définir la culture urbaine (Grafmeyer et Joseph, 1990) la sociologie urbaine, notamment pour concevoir l'urbanisation (Ostrowetsky, 1996) distincte de l'urbanité (Laborit, 197), l'espace comme espace social (Castells, 1981), l'espace urbanisé comme résultant de la mobilité (Rémy et Voyé, 1992 ; Rémy et Leclercq, 1998), pour questionner les processus de ségrégation et de mise à distance (De Rudder, 1995); dans la théorie des attitudes pour envisager la dimension attitudinale comme méthode de recueil (Fishbein et Ajzen, 1975; Lambert et alii, 1960 ; Lambert, 1967) ; dans l'anthropologie urbaine pour référer à des méthodes d'investigations directes (Raulin, 2002) ; dans l'analyse du discours pour aller au-delà des faits de surface et appréhender le non-dit comme un des éléments structurant des stéréotypes sur l'urbanité (Guespin, 1971, 1976), pour travailler sur les aspects mémoriels des discours tenus (Sériot, 1994 ; Paillard, 1994) ; dans la socio-sémiotique de l'espace pour définir l'épaisseur identitaire et surtout la dynamique produisant les espaces et territoires (Lévy, 1996); dans la géographie sociale pour définir le territoire dans son rapport aux espaces perçus et/ou vécus ; (Tizon, 1996 ; Di Méo, 1990) pour envisager le marquage des espaces (Veschambre, 2006) et les rapports entre appropriation des espaces et légitimation des pouvoirs (Ripoll et Veschambre, 2002) ; dans le dialogisme pour à la fois cadrer théoriquement l'interdiscursivité et à la fois pour disposer d'outils d'analyse des données (Bakthine, 1977 et 1995; Peytard, 1990 ; Barbéris, 2005) ; dans la géographie urbaine dans la mesure où les dénomination et désignation des entités urbaines questionnent d'évidence la mise en mots de l'espace (Vauvour-Jouve, 2001) et de la territorialité ainsi que la rencontre avec l'habitant/l'habiter mythique (Roncayolo, 1997); l'anthropologie de l'espace pour ses réflexions sur la délimitation, les frontières et les limites (Paul-Lévy et Segaud, 1983); et enfin dans les Sciences de l'information pour la conception de la ville comme territoire d'appartenance politique et de gestion des flux d'informations (Lamizet, 2000).

Une telle liste ne prétend pas à l'exhaustivité mais souhaite seulement rendre compte de l'arrière-plan conceptuel qui a prévalu à la conceptualisation sociolinguistique du champ urbain. Dès lors, et pour saisir le terme même de centralité sociolinguistique mis en regard avec celui de nivellement sous l'angle de la spatialité, restent à définir quelques derniers concepts de base.

\section{Les concepts nécessaires pour aborder les tensions et les conflits}

Ainsi, faire de la sociolinguistique urbaine c'est bien entendu faire de la sociolinguistique, mais en interrogeant plus précisément en rapport avec les parlures urbaines a) d'une part les liens entre la production discursive des espace(s) et la mise en mots des lieu(s) et, d'autre part b) Les déplacements, les frontières et les limites intra-urbaines pour ce qu'ils permettent de saisir l'identité socio-langagière et les tensions et les conflits à l'intérieur de la communauté urbaine. C'est ainsi tenter de faire valoir minimalement l'opérativité tant discursive que 
cognitive de deux processus concomitants relatifs aux formes dites et/ou perçues comme spécifiques à un espace urbain donné :

a) l'évaluation (hiérarchiser socialement les variétés de langues et les langues) et

b) l'identification (attribuer les formes à des portions d'espaces) en tant qu'ils concourent à produire le-dit espace, à l'organiser tout autant que ce que l'on appelle les structures socio-spatiales.

Le socle conceptuel minimal visant à rendre compte de ces processus centraux à l'intelligibilité de l'espace des villes tient en trois termes.

L'identité urbaine. Le concept permet de rendre compte des pratiques langagières des locuteurs urbains se représentant la tension ainsi posée entre leur indispensable identification à une communauté et leur propre différenciation par rapport à d'autres lieux communautaires de tous ordres, signalant une appartenance groupale; par la prise en compte et l'analyse de leur mise en mots de cette tension, il s'agit de dégager la spécificité identitaire de toute ville, et partant de tout espace urbanisé.

La mobilité spatio-linguistique. L'un des effets inhérents à la mobilité spatiale est de mettre à distance les individus et les groupes, et de recomposer le lien social autour notamment des représentations que l'on s'accorde sur autrui et sur soi-même. Plus l'espace est urbanisé, plus le rapport à l'autre, le rapport à sa façon de parler, fonde les limites et frontières intra-urbaines ; de même le discours sur autrui, sur la langue ou la pratique de langue d'autrui devient par défaut autrui. Ainsi le concept ne renvoie pas au seul changement (ou la volonté mise en mots de changement) de langue ou de variété qui accompagnerait une mobilité sociale généralement ascendante, mais à la façon dont les déplacements que l'on opère et les rencontres «langagières » que l'on fait (ou croit faire d'ailleurs) déterminent la représentation que l'on croit commune de la ville que l'on habite; il rend compte de la mise en contact différenciée temporellement et spatialement "de groupes urbains posés comme distincts par les acteurs de la mobilité spatiale » (Bulot, 1999 : 43).

Et enfin la territorialisation linguistique. La mise en mots de l'espace urbanisé (c'est-à-dire où le fait de pouvoir assumer ou non (Ripoll et Veschambre, 2005) la survalorisation de la mobilité spatiale (Rémy et Voyé, 1992)) relève d'une double détermination : celle relative au territoire dans la mesure où on doit considérer l'espace comme une aire légitime de proxémie liée aux parcours, aux lieux de vie, de sociabilité et celle relative à la territorialité que l'on peut concevoir comme la représentation de ce même territoire. Ainsi le concept interroge le terrain pour savoir s'il y a juxtaposition, coïncidence entre deux univers représentationnels, entre un lieu tel qu'il est dit et les représentations topolectales de la langue. La territorialisation linguistique ${ }^{32}$ est la façon dont, en discours, les locuteurs d'une ville s'approprient et hiérarchisent les lieux en fonction des façons de parler (réelles ou stéréotypées) attribuées à eux-mêmes ou à autrui pour faire sens de leur propre identité.

\section{LA SPATIALITÉ URBAINE COMME UNE MISE EN MOTS}

\section{Pour discriminer les espaces de ville : citadin, urbain et urbanisé}

La spatialité en sociolinguistique urbaine est définie comme une entité méthodologique doublement articulée sur d'une part l'espace (comme aire symbolique, matérielle qui inscrit l'ensemble des attitudes et des comportements langagiers ou non dans une cohérence globale, communautaire) et d'autre part le lieu (en tant que repère concourant à la sémiotisation sociale

\footnotetext{
32 Bulot, T. (2006). Espace urbain et mise en mot de la diversité linguistique. Dans : Les codes de la ville (Cultures, Langues et formes d'expression urbaines. Paris : L'Harmattan (collection Espaces Discursifs)/ Stauffenburg Verlag, pp. 9-28.
} 
et sociolinguistique de l'aire géographique citadine) ${ }^{33}$. De fait, cette spatialité (Figure 8) émerge de la projection des traits locatifs (c'est-à-dire relatifs aux lieux) produits en discours sur les espaces sociaux : les locuteurs ont le sentiment d'identifier ${ }^{34}$ un espace géographique (et non un espace social) car ils le définissent comme un lieu; elle émerge également de la projection des traits spatiaux discursivisés sur les lieux : effectivement les locuteurs décrivent les lieux comme s'il s'agissait d'espaces sociaux.

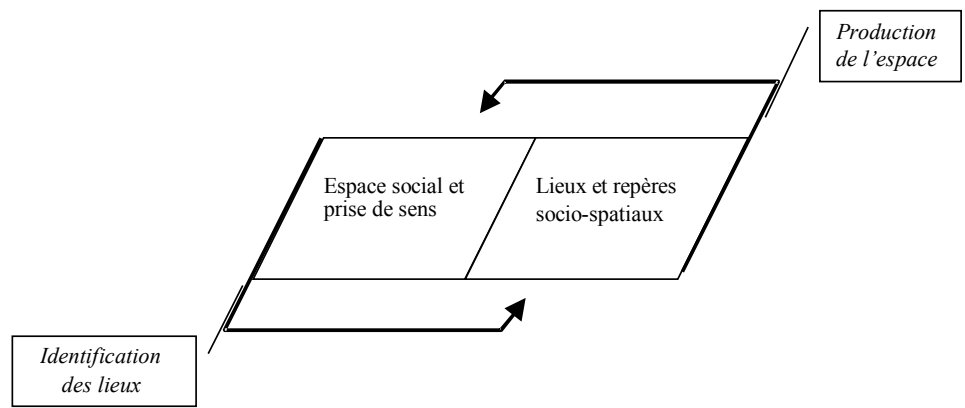

Figure 8: La spatialité urbaine mise en mots (Bulot, 2004a : 117).

$\mathrm{Au}$ regard des travaux sur l'espace et sur les lieux (notamment ceux de la géographie sociale) et des résultats de nos enquêtes sociolinguistiques ${ }^{35}$, est apparue la nécessité de distinguer trois termes pour rendre compte au plus près de la diversité discursive des espaces «de ville »: il s'agit de différencier les espace citadin, espace urbain et espace urbanisê $e^{36}$. Une telle taxinomie se conçoit en parallèle avec une première distinction (Cauvin, 1999) opérée pour discriminer ce que la vulgate peut rapidement percevoir : tout individu et tout groupe social a des usages divers et diversifiés de l'espace qu'il occupe et/ou produit d'une part, et, d'autre part, que la notion empirique d'espace urbain recouvre des réalités sociales, géographiques, interactionnelles (Mondada, 2000 et 2004) qui mène à en percevoir la complexité. La géographie sociale distingue effectivement quatre types d'espaces interreliés dont nous aménageons les définitions.

1. un espace chorotaxique qui constitue l'ensemble des unités reconnues comme distinctes par le sujet : il est perçu comme objectif dans la mesure où les désignations qui le concernent sont des lexèmes hyperonymiques des réalités observables (bâtiment, rue, commerce,...).

2. un espace chorotaxique change en fonction des activités qui lui sont attribuées. Il devient un / des espace(s) fonctionnel(s) dans la mesure des attributs possibles (dont langagiers) retenus pour les lieux qui le/ les caractérisent et pour la nature des liens posés entre les lieux.

3. les espaces fonctionnels sont vécus comme des espaces d'action parce qu'ils ancrent le sujet sur un réel où leurs actions et leur discours prennent sens au regard de la communauté. C’est l'espace de la légitimité des pratiques.

4. les espaces cognitifs sont les entités qui inscrivent le sujet dans la perception de la justesse de ses représentations; ils sont les espaces fonctionnels reconnus par un individu, et manifestent la dimension praxique du discours et des représentations du réel. C'est l'espace légitime mis en mots.

\footnotetext{
33 Cf. Bulot (2004a).

${ }_{34}$ À poser comme le processus visant à attribuer une façon de parler à un espace donné et à la reconnaitre comme constitutive de sa reconnaissance.

35 Voir Bulot (1999 et 2004b) ainsi que Bulot, T. (2001). Ségrégation et urbanisation linguistique : l'altérité urbaine définie ou 'l'étranger est une personne'. Diverscité Langues, VI. (http://www.teluq.uquebec.ca/diverscite) Québec : Télé Université du Québec, 21 pages.

${ }^{36}$ Pour continuer la glose sociolinguistique de l'urbanisation.
} 
Sont ainsi posés, pour une approche socio-langagière de la spatialité urbaine trois concepts qui renvoient chacun à des processus distincts mais inter-reliés dans la mesure où ils réfèrent à des spatialisations perçues comme homogènes. On va ainsi parler d'un espace citadin lorsque il s'agit d'exprimer la part descriptive et circonscriptible d'un espace que tous les locuteurs doivent s'accorder à reconnaitre comme tel; il procède conjointement des espaces chorotaxiques (dans la mesure des catégories le désignant) et des espaces d'action (par la nécessité de concevoir les catégories comme liées aux pratiques langagières). On va également parler d'un espace urbain pour révéler la résultante du rapport entre l'espace chorotaxique et la somme des diverses interactions entre les espaces fonctionnels et les espaces d'action. Un espace urbain se situe précisément sur une confusion nécessaire entre un réel objectivé (les catégories citadines) et des pratiques discursives tendues ${ }^{37}$ au sein de la communauté sociale. Il faut garder à l'esprit que la mobilité (sociale et linguistique) inscrite dans les valeurs et les comportements induit une production discursive de l'espace communautaire où la dialectique de l'identification et de la différenciation joue à plein: le sentiment d'appartenance communautaire (c'est-à-dire à un espace urbain spécifique) n'a de valeur que s'il permet aux groupes de se différencier dans la même communauté. Et enfin, on va parler d'un espace urbanisé pour introduire l'idée de la confusion des différents types d'espaces, confusion donnant à voir les espaces citadin ou urbain comme des données objectivées tantôt par les catégories chorotaxiques, tantôt par les comportements observables des usagers de ces espaces. Cet espace est non seulement nécessairement lié à la perception sociale de l'espace communautaire mais plus encore à la représentation dominante et aux discours en relevant; il est lié à la perception sociale d'un modèle culturel urbain exacerbant, sur une aire géographique réduite (celle de la communauté citadine), la mesure de la distance sociale par la mise en mots de la distance linguistique.

C'est ce dernier type d'espace qui constitue l'objet scientifique de la sociolinguistique urbaine dans la mesure où il justifie au moins l'éthique de l'engagement que nous évoquions ci-dessus. Mais - et davantage - cette conceptualisation permet de saisir la spécificité de l'approche: on ne peut faire valoir la prégnance du langagier, du discursif sur l'espace commun d'une ville sans concevoir 1) que celui-ci est un processus social situé dans des interactions diverses, pour des acteurs différents, et 2) que par conséquent il est à la fois perçu et vécu comme homogène et comme hétérogène et 3) que la distance, construite comme toujours spatiale, permet l'établissement d'une altérité urbanisée ("d'où est l'autre ?» et, partant, "d'où suis-je?») assurant, entre autres, la gestion discursive de l'identification et de la différenciation des locuteurs de l'espace évalué et décrit; en effet, confronté aux représentations sociolinguistiques, ce processus identificatoire, lié a priori aux seuls lieux et aux seuls espaces, introduit a posteriori la confusion entre la distance géographique (alors mise en mots pour couvrir la réelle volonté de distance sociale d'une population donnée) et distance sociale sur fond d'une distance linguistique objectivée.

\section{Modéliser la spatialité urbanisée : la dénomination}

En tant que processus spécifiquement dénominatif et par ailleurs absolument identitaire, la territorialisation se décompose en trois temps (voir Figure 9) liés et systématiques (mais qui ne sont pas nécessairement impartis à des repères identiques) que sont la circonscription (la mise en place discursive des limites), la définition (la mise en mots des attributs définitoires) et la production (la mise en place des frontières). Cette approche permet de comprendre comment est gérée la tension inhérente à tout espace de ville entre la nécessité d'appartenir à une communauté unifiante et l'exigence de pouvoir se différencier: à partir de repères apparemment objectifs car relatifs au bâti citadin (les dénominations du citadin : les lieux

\footnotetext{
${ }^{37}$ Ces pratiques sont dites 'tendues', car, bien qu'elles n'aboutissent pas nécessairement à un conflit, elles semblent émaner d' imaginaires contradictoires et de pratiques sociales antynomiques.
} 
disants), les différents acteurs/locuteurs de la ville objectivent les attributions socio-langagières en les transposant sur l'urbain (les dénominations urbaines: les lieux dits), pour au final produire un discours significatif des tensions urbaines et constitutif de l'espace ainsi urbanisé (les dénominations urbanisées : les lieux de ville) ${ }^{38}$.

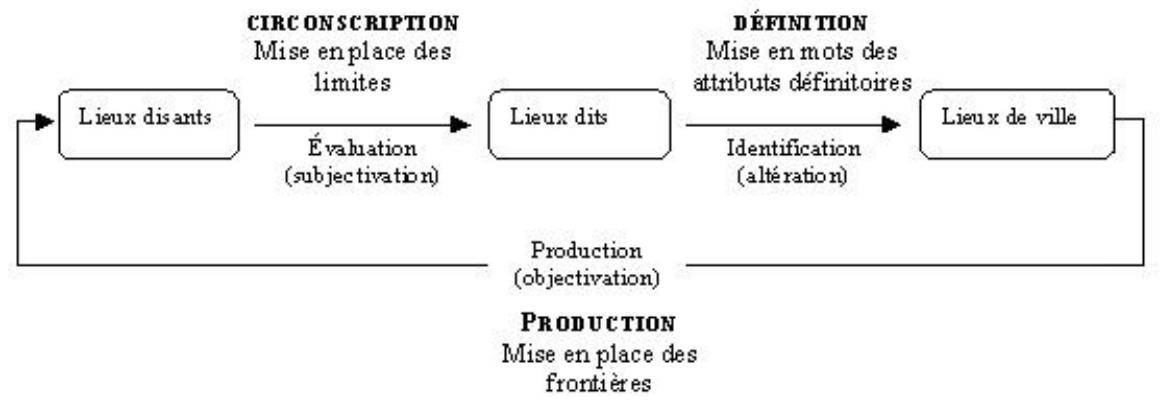

Figure 9. La territorialisation (socio)linguistique (Bulot, 2004b : 114).

Cela posé, il est ainsi possible de reformuler les propositions initiales concernant les trois types d'espaces : l'espace citadin est caractéristique de l'existence de dénominations objectivées qui permettent de penser produire en discours un espace identique pour tous les locuteurs et habitants par le partage de catégories communes. L'espace urbain est lui caractéristique de dénominations perçues comme objectivées mais qui renvoient sans que cela soit vécu comme tel à l'appartenance sociale du locuteur qui émet la dénomination (les termes locatifs stigmatisent ou valorisent les locuteurs via les parlures identifiées). Et enfin l'espace urbanisé (fondé sur la confusion entre les deux premiers types d'espace) est caractéristique de dénominations potentiellement perçues (les représentations) comme objectivées, mais de fait vécues (les pratiques) comme renvoyant à l'appartenance sociale du locuteur qui émet la dénomination et comme exprimant les rapports hiérarchisés quant à ces trois niveaux: l'espace, le social et les langues et parlures. Les distances sociale et linguistique sont ainsi au cœur des tensions...

Sachant que l'on cherche, ici, à modéliser les corrélations entre les niveaux spatiaux, sociaux et linguistique qui spécifient l'espace urbanisé (E), ces propositions, qui demeurent discutables, peuvent être ainsi représentées :

Soit l'axiomatique suivante ${ }^{39}$ $\mathrm{e}=$ est l'espace citadin (les dénominations objectivées vécues et perçues comme renvoyant à l'espace commun).

$\varepsilon=$ est l'espace urbain (les dénominations perçues comme objectivées et renvoyant à l'appartenance sociale).

$\mathrm{P}=$ sont les pratiques socio-discursives (dénominations vécues) et se décline en $\mathrm{P}_{\mathrm{a}}$ (le discours sur la pratique) $+\mathrm{D}_{\mathrm{i}}$ (la pratique du discours)

$\mathrm{D}=$ est la distance et se décline en $\mathrm{d}^{\mathrm{s}}$ (distance sociale) $+\mathrm{d}^{\mathrm{l}}$ (distance linguistique)

Quel est E (l'espace urbanisé) ? Nous raisonnons en trois temps.

\footnotetext{
${ }^{38}$ Pour des définitions commentées des termes «lieux-dits», « lieux-disants » et « lieux de ville », voir Bulot (2004a) ; pour la distinction des termes «limite» et «frontière», voir Bulot (2004b) ; pour la distinction entre «identification » et « évaluation », voir Bulot et Bauvois (1998).

39 « = » veut dire en quelque sorte « se réécrit», se compose d'éléments constitutifs, nécessaire et corrélés. « - » ou «+» veulent exprimer les corrélations négative / positive, c'est à dire soustractives ou additionnelles des facteurs corrélés. «() » veut exprimer la relation de corrélation. Ce qui est mis en indice ou en exposant signifie « relatif à » l'entité ou le processus nommés. Merci à Gauthier Bulot pour son aide technique à la présente formalisation.
} 
1. Si l'espace citadin renvoie à des dénominations qui ne sont pas marquées en discours comme spécifiques d'une hiérarchie sociale des espaces $\left(\varepsilon-\mathrm{P}^{\varepsilon}\right)$, et si l'espace urbain se caractérise par de telles dénominations certes objectivées mais marquées par la-dite hiérarchie $\left(\mathrm{e}+\mathrm{P}^{\mathrm{e}}\right)$, alors l'espace urbain est de fait issu des corrélations entre l'absence de pratiques discursives perçues comme situées socialement mais effectives dans la description de l'espace commun $\left(\left(\varepsilon-\mathrm{P}^{\varepsilon}\right)+\mathrm{P}^{\mathrm{e}}\right)$

$$
\text { Si e }=\left(\varepsilon-\mathrm{P}^{\varepsilon}\right) \text { et } \varepsilon=\left(\mathrm{e}+\mathrm{P}^{\mathrm{e}}\right) \text { alors } \varepsilon=\left(\varepsilon-\mathrm{P}^{\varepsilon}\right)+\mathrm{P}^{\mathrm{e}}
$$

2. Si les pratiques socio-discursives sont dans un constant décalage - par hypothèse du moins - entre la pratique du discours et le discours sur la pratique, alors il faut admettre qu'un tel décalage concerne tout autant l'espace citadin que l'espace urbain : l'un et l'autre sont la résultante de cette double détermination.

$$
\text { Si } \mathrm{P}=\mathrm{P}_{\mathrm{a}}+\mathrm{D}_{\mathrm{i}} \text { alors } \mathrm{P}^{\mathrm{e}}=\left(\mathrm{P}_{\mathrm{a}}+\mathrm{D}_{\mathrm{i}}\right)^{\mathrm{e}} \text { et } \mathrm{P}^{\varepsilon}=\left(\mathrm{P}_{\mathrm{a}}+\mathrm{D}_{\mathrm{i}}\right)^{\varepsilon}
$$

3. Si l'espace urbanisé (E) procède des deux premiers types d'espaces (les catégories citadines et les catégories urbaines), il est perçu comme un espace urbain $\left(\varepsilon-\left(\mathrm{P}_{\mathrm{a}}+\mathrm{D}_{\mathrm{j}}\right)^{\mathrm{q}}\right)$ mettant en mots $\left.\left(\left(\mathrm{P}_{\mathrm{a}}+\mathrm{D}_{\mathrm{i}}\right)^{\mathrm{g}}\right)\right)$ les catégories citadines, déterminée par une double corrélation entre la distance sociale et la distance linguistique $\left(\sqrt{ }\left(\mathrm{d}^{\mathrm{s}^{2}}+\mathrm{d}^{12}\right)\right)$ mises en mots ${ }^{40}$.

$$
\begin{aligned}
\text { Si } E= & \left(\left(\varepsilon-\left(\mathrm{P}_{\mathrm{a}}+\mathrm{D}_{\mathrm{i}}\right)^{\varepsilon}\right)+\left(\mathrm{P}_{\mathrm{a}}+\mathrm{D}_{\mathrm{i}}\right)^{\mathrm{e}}\right)+\sqrt{\left(\mathrm{d}^{\mathrm{s}^{2}}+\mathrm{d}^{1^{2}}\right)^{\mathrm{p}}} \\
& \text { Alors } \mathrm{E}=\left(\left(\varepsilon-\mathrm{P}^{\varepsilon}\right)+\mathrm{P}^{\mathrm{e}}\right)+\sqrt{ }\left(\mathrm{d}^{\mathrm{s}^{2}}+\mathrm{d}^{1^{2}}\right)^{\mathrm{p}}
\end{aligned}
$$

Nous avons parfaitement conscience qu'une telle formalisation d'un modèle initialement «discursif» n'a de sens que si le modèle ainsi formalisé apporte une aide à la réflexion générale ; il semble que c'est le cas dans la mesure où une seule manipulation de l'équation permet

a) de montrer qu'il est théoriquement possible de mettre en mots la distance

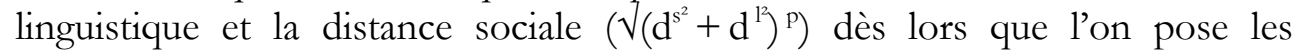
catégories citadines comme étant le seul réel observable,

$$
\left.E-\left(\left(\varepsilon-\left(P_{a}+D_{i}\right)^{\varepsilon}\right)+\left(P_{a}+D_{i}\right)^{e}\right)\right)=\sqrt{ }\left(d^{s^{2}}+d^{1^{2}}\right)^{p}
$$

et

b) que ces distances ainsi corrélées l'une à l'autre, dissociées des pratiques socio-langagières liées à l'espace urbanisé, autrement dit à la territorialisation, sont de fait le nivellement linguistique. Cela si ce concept finit par faire affirmer ou accroire que les locuteurs de langue française et habitant un espace francophone parlent la même langue - le français -, en oubliant, ainsi, qu'ils font actes langagiers et identitaires de variétés qui fonctionnent comme des langues, voire qui sont des langues pour ces mêmes locuteurs.

$$
\sqrt{ }\left(d^{s^{2}}+d^{1^{2}}\right)^{p}=E-(e+P)
$$

\section{Le confinement linguistique}

Nous avons annoncé - et d'ailleurs déjà commenté - dans notre titre général le terme de centralité linguistique; ce concept a un défaut majeur qui provient des représentations qui prévalent sur la ville dans les travaux des sociolinguistes au moins. Il présuppose, qu'à l'instar des centres-villes qui ne sauraient qu'être uniques pour chaque ville, la seule et unique norme

\footnotetext{
${ }^{40}$ Cette formule ( racine carrée de la somme des carrés ») signifie que la somme des valeurs est différente de la racine de la somme des carrés. Autrement dit chacune des valeurs reste entière même si chacune contribue à produire une troisième valeur.
} 
de référence va se trouver localisée en discours en un seul espace, celui qui grosso modo est l'espace historique, culturel, patrimonial de la cité. Il convient de parler de centralité sociolinguistique dès lors que le concept renvoie à la territorialisation qui nous le verrons dans les études de cas, ne peut être que multiple au sein même d'une seule communauté urbaine.

Rappelons que la territorialisation ${ }^{41}$ procède d'évidence des stratégies identitaires (Camilleri, 1996) urbanisées et c'est pourquoi elle donne à percevoir la polymorphie de l'identité urbaine - sous l'angle des divers types d'espaces proprement relatifs à ce que les locuteurs identifient comme leur ville. Davantage, elle permet de concevoir via la mise en mots différenciée et située des parlures que les espaces sont:

a) normatifs mais différemment partagés, espaces que les rapports à la dominance imposent à la communauté ainsi que des espaces

b) singuliers et différenciateurs dans la mesure où le locuteur collectif instauré use différemment de la confusion idéologique entre les espaces citadins, urbains et urbanisés.

Ainsi, le partage d'un espace de référence (où le discours visant à poser ce partage comme effectif sans qu'il le soit, à poser et espace comme unique sans qu'il le soit, etc.) donne à construire une représentation du partage d'une norme de référence; donne à dire son attachement à la norme linguistique pour faire état de son attachement nécessaire à un espace identitaire (cf. Cauvin, op. cit.) donc commun mais qui n'est ni nécessairement continu, ni aussi vaste pour tous; enfin pour donner sens et légitimité à ses actes.

Non seulement la centralité ne renvoie pas à un seul centre, un seul point nodal, mais à une pluralité de "centres", mais encore chacun d'eux peut-être perçu voire vécu comme hiérarchisé ou non hiérarchisé selon qu'un discours existe ou non le ou les spécifiant. Rapporté aux langues et aux variétés, un tel processus questionne évidemment ce que le discours suppose - c'est-à-dire celui posant un nivellement permanent et récurrent de la langue - de renforcement de la minoration sociale. Ce sont par exemple les cas de confinement linguistique qui assurent à chacun des systèmes en contact - tant spatiaux que sociolinguistiques - une part identitaire, identificatoire et donc perçue comme une catégorisation somme toute positive mais qui hésite entre l'élargissement de l'espace communautaire à la totalité de la ville, et une différenciation articulée aux pratiques ségrégatives.

Autrement dit, la constitution d'un espace de référence perçu comme homogène implique l'existence d'un contre-espace de référence. Les espaces ne sont pourtant ni homogènes ni parfaitement partagés par l'ensemble des locuteurs dans la mesure où les logiques d'appropriation et / d'assignation (Ripoll, 2006) ne le sont pas davantage. De la sorte, il faut concevoir que chacun des types d'espace, et partant des variétés et/ ou des langues et / ou des normes y afférant, ne peut se concevoir que comme un ensemble multipolaire.

\section{CONCLUSION : MULTIPOLARISATION DES ESPACES ET DISCRIMINATION DES}

\section{NORMES}

Nous ne voulons pas reprendre la réflexion sur la notion même de discrimination en sociolinguistique ${ }^{42}$ mais souligner, par le titre que nous donnons à cette conclusion en tous points provisoire d'une théorisation en mouvement, que l'entrée par le discours sur l'espace langagier est d'une efficacité certaine lorsqu'il s'agit de constater voire de mesurer les effets praxiques du discours sur les langues et leurs espaces d'une part, et, d'autre part, sur la mise en mots perçue de la diversité linguistique. De fait, si l'on peut percevoir que nombre de

\footnotetext{
${ }^{41}$ Voir Bulot (2004b) pour le concept.

42 Voir la référence citée note 10.
} 
locuteurs déclinent leur identité linguistique eu égard à une communauté linguistique de référence, il apparaît également que ce rapport - certainement dialectique - n'est pas anthropologiquement considéré autrement perçu que dans la relation à leur espace d'action. Ainsi, de même que les espaces sont multipolarisés dans la mesure de leur détermination au social, les langues et ou les/leurs variétés échappent nécessairement tout ou partie à une standardisation voire à un nivellement qui est de fait plus idéologique dans ses formes d'expression que strictement pratique. Il semble bien que la dimension auto-gestionnaire des pratiques glottopolitiques ait pour finalité de rendre compte du processus visant à discriminer les normes et, partant, à rendre sans cesse possible et effective l'émergence d'autres langues ${ }^{43}$ voire d'autres normes.

\section{thierry.bulot@free.fr ou thierry.bulot@uhb.fr}

\section{RÉFÉRENCES}

Andersen, H. (1988). Centre and periphery : adoption, diffusion and spread. In Historical dialectology. Regional and Social. Berlin/ New York/ Amsterdam: Mouton de Gruyter, pp. 39-83.

Bachmann, C. et Simonin, J. (1993). Le social comme on le parle. Actions et Recherches Sociales, 2, pp.6579 .

Baggioni, D. (1994). Les langues dans l'espace urbain à l'Ile Maurice ». Dans : J.M. Barbéris (Dir.), La ville (Arts de Faire et Manières de Dire. Montpellier : Paxiling, pp. 137-162.

Bakhtine, M. (1977). Le marxisme et la philosophie du langage, Éditions de Minuit, Paris.

Bakhtine, M. (1995). Esthétique et théorie du roman, Réed.,1978, Gallimard, Paris.

Barbéris J.-M. (Dir.) (1994).La ville (Arts de Faire et Manières de Dire, Paxiling, Montpellier.

Barbéris J.-M. (2005). La ville et ses composantes : l'émergence des catégories en interaction orale. Revue de l'université de Moncton, 36/1, pp. 31-59.

Boyer, H. (2003). De l'autre côté du discours (Recherches sur les représentations sociolinguistiques). Paris: L'Harmattan.

Bulot, T. (Dir.) (1999). Langue urbaine et identité (Langue et urbanisation linguistique à Rouen, Venise, Berlin, Athènes et Mons). Paris: L'Harmattan.

Bulot, T. (2001a). La construction de la référence communautaire : le français de référence au centreville. Cabiers de l'Institut de Linguistique de Louvain, 27 / (1-2), pp. 35-42.

Bulot, T. (2001b). L'essence sociolinguistique des territoires urbains : un aménagement linguistique de la ville ?. Cabiers de Sociolinguistique, 6, pp. 5-11.

Bulot, T. (2003a). Matrice discursive et confinement des langues : pour un modèle de l'urbanité. Cabiers de Sociolinguistique, 8, pp. 99-110.

Bulot T. (2003b). Le discours sur l'identité ethnolinguistique : identifier pour se définir en contexte diglossique. Dans: Textes et Contextes Culturels. Mont Saint Aignan: Publications de l'Université de Rouen, 11 pages (A paraittre en 2006).

Bulot, T. (2004a). La double articulation de la spatialité urbaine : «espaces urbanisés » et «lieux de ville » en sociolinguistique. Dans: T. Bulot (Dir.), Lieux de ville et identité (perspectives en sociolinguistique urbaine). Paris: L'Harmattan (Collection Marges Linguistiques), pp. 113-146.

Bulot, T. (2004b). Les frontières et territoires intra-urbains : évaluation des pratiques et discours épilinguistiques. Dans : F. Fusco (Dir.), Le città plurilingui. Lingue e culture a confronto in situarioni urbane / Multilingual cities. Perspectives and insights on languages and cultures in urban areas, Udine : Forum Editrice Universitaria Udinese srl, pp. 110-125.

${ }^{43}$ Elles ne sont pas nécessairement nouvelles du point de vue de leur structure, mais tout à fait autre quant à leur fonction. 
Bulot, T. (2005). Que parle-t-on en Pays de Caux ?. Émergence et/ou continuité d'une communauté sociolinguistique régionale. Marges Linguistiques, 10. Saint-Chamas: http://www.margeslinguistiques.com/, pp. 88-117.

Bulot, T. et Bauvois C. (1998). Le sens du territoire (l'identification géographique en sociolinguistique). Revue PArole, 5/6. Mons : Université de Mons Hainaut, pp. 61-80.

Bulot, T. et Veschambre V. (2006). Sociolinguistique urbaine et géographie sociale: articuler l'hétérogénéité des langues et la hiérarchisation des espaces. Dans Dire et faire la géographie sociale. Rennes : Presses Universitaire de Rennes, (sous presse).

Calvet, L.-J. (1994). Espace urbain et plurilinguisme. Dans : J.M. Barbéris (Dir.), La ville (Arts de Faire et Manières de Dire. Montpellier, : Paxiling, pp. 163-174.

Calvet, L.-J. (2004). La sociolinguistique et la ville. Hasard ou nécessité ?. Dans : T. Bulot (Dir.), Lieux de ville et identité (perspectives en sociolinguistique urbaine). Paris: L'Harmattan (Collection Marges Linguistiques), pp. 13-29.

Camilleri, C. (1996). Stigmatisation et stratégies identitaires. Dans : La ville : agrégation et ségrégation sociales. Paris: L'Harmattan, pp. 85-92.

Castells, M. (1981). La question urbaine. Paris : Maspéro/Fondations.

Cauvin, C. (1999). Propositions pour une approche de la cognition spatiale intra-urbaine. Cybergéo, 72. (http://www.cybergeo.presse.fr/geocult/texte/cognima.htm.).

Dauzat, A. (1906). Essai de méthodologie linguistique dans le domaine des langues et des patois romans, Champion, Paris.

De Rudder, V. (1995). La ségrégation est-elle une discrimination dans l'espace. Dans : R. Galissot et J.M. Moulin (Dirs), Les quartiers de la ségrégation (Tiers monde on Quart monde ?). Paris : Editions Karthala, pp. 11-30.

Di Méo, G. (1990). De l'espace vécu aux formations socio-spatiales. Géographie Sociale, 10. Caen: Presses Universitaires de Caen, 13-23.

Eloy, J.-M. (2004). Des langues collatérales : problèmes et propositions. Dans : J-M. Eloy (Dir.), Des langues collatérales. Paris: L'Harmattan (Collection Espaces Discursifs), pp. 5-25.

Fishbein, M., Ajzen, I. (1975). Belief, Attitude, Intention and Behaviour. An Introduction to Theory and Research. Addison-Wesley: Reading, Mass.

Gasquet-Cyrus, M. (2004). Sociolinguistique urbaine ou urbanisation de la sociolinguistique ? Regards critiques et historique sur la sociolinguistique.. Dans : T. Bulot (Dir.), Lieux de ville et identité (perspectives en sociolinguistique urbaine). Paris: L'Harmattan (Collection Marges Linguistiques), pp. 31-70.

Grafmeyer, Y., Joseph, I. (1990). L’École de Chicago (Naissance de l'écologie urbaine). Paris : Aubier.

Guespin, L. (1976) : Introduction. Langages, 41, pp. 47-78.

Guespin, L. (Dir.) (1971) : Le discours politique. = Langages 23,. Paris : Didier-Larousse.

Guy, C., Givord, L. (2004). Rennes, le pari d'une agglomération multipolaire. Paris : L'aube.

Labov, W., 1976, Sociolinguistique. Paris : Les Éditions de Minuit.

Labov, W. (2002). Qu'est-ce qu'un fait linguistique ?. Dans : M. Santacroce (Dir.), Faits de langue, faits de discours. Paris: L'Harmattan (Collection Marges Linguistiques), pp. 11-118.

Laborit, H. (1971). L'Homme et la ville. Paris, Flammarion.

Lambert, W.E., 1967, The social psychology of bilingualism. Journal of Social Issues, 23, 91-109.

Lambert, W.E., Hodgson, R., Gardner, R.C. (1960). Evaluational reactions to spoken languages. Journal of Abnormal and Social Psychology, 60, 44-51.

Lamizet, B. (2000). Le sens de la ville. Paris : L'Harmattan.

Lévy, A. (1996). Pour une socio-sémiotique de l'espace. Problématique et orientations de recherche. Dans : S. Ostrowetsky (Dir.), Sociologues en villes. Paris: L'Harmattan, pp. 159-177.

Lodge, A. (1999). Convergence and divergence in the developpement of the Paris urban vernacular. Sociolinguistica, 13, 51-68.

Manessy, G. (1990). Mode de structuration des parlers urbains. Dans: Des langues et des villes, Paris: Didier-Érudition, pp. 7-27. 
Manzano, F. (1997). Le gallo à la fin du XX $\mathrm{XX}^{\mathrm{eme}}$ siècle : mythes, réalités et perspectives. Cabiers de Sociolinguistique, 2-3, pp. 405-451.

Marcellesi, J.-B., Lozay, G. (1983), Les étudiants de l'université de Haute-Normandie et le .problème du dialecte : premiers résultats d'une enquête. Cabiers des Annales de Normandie, 14, pp. 193-197.

Mensire, R., 1984 (1946), Le Pays de Caux «son origine, ses limites, son histoire. Yvetot: Éditions de l'imprimerie commerciale d'Yvetot.

Mondada, L. (2000). Décrire la ville (La construction des savoirs urbains dans l'interaction et dans le texte), Paris : Anthropos.

Mondada, L. (2004). La ville n'est pas peuplée d'êtres anonymes : processus de catégorisation et espace urbain. Dans: T. Bulot (Dir.), Lieux de ville et identité. Paris: L'Harmattan (Collection Marges Linguistiques), pp. 71-111.

Ostrowetsky, S. (1996). Sociologues en ville : introduction. Dans : S. Ostrowetsky (Dir.), Sociologues en villes. Paris: L'Harmattan, pp. 9-17.

Paillard, D. (1994). Russie/URSS : le discours national russe comme mémoire du refus. Langages, 114, 98-108.

Paul-Lévy, F., Segaud, M. (1983). Anthropologie de l'espace. Paris : Centre de Création Industrielle/ Centre Georges Pompidou.

Peytard, J. (1990). Évaluation sociale dans les thèses de Mikhail Bakhtine et représentations de la langue. Langue Française, 85, pp. 6-21.

Raulin, A. (2002). Anthropologie urbaine. Paris : Armand Colin.

Rémy J., Leclercq, É. (1998). Sociologie urbaine et rurale (L'espace et l'agir). Paris : L’Harmattan.

Rémy, J., Voyé, L. (1992). La ville : vers une nouvelle définition? Paris : L’Harmattan.

Ripoll, F. (2006). Réflexions sur les rapports entre marquage et appropriation de l'espace. Dans : T. Bulot et V. Veschambre (dirs.), Mots, traces et marques (Dimensions spatiale et linguistique de la mémoire urbaine). Paris: L'Harmattan (Collection Espaces Discursifs), pp. 15-36.

Ripoll, F., Veschambre V. (2002). Face à l'hégémonie du territoire : éléments pour une réflexion critique. Lire les territoires, 3, 261-288.

Ripoll, F., Veschambre V. (2005). Sur la dimension spatiale des inégalités : contribution aux débats sur la 'mobilité et le capital spatial'. Dans : Rural-Urbain. Nouveaux liens, nouvelles frontières. Rennes: Presses Universitaires de Rennes, pp. 467-483.

Roncayolo, M. (1997). La ville et ses territoires. Paris : Folio-Essais (nouvelle édition revue).

Sériot, P. (1994). Le cas russe: Anamnèse de la langue et quête identitaire (la langue-mémoire du peuple). Langages, 114, pp. 84-97.

Tizon, P. (1996). Qu'est-ce que le territoire ?. Dans : G. Di Méo (Dir.), Les territoires du quotidien. Paris : L'Harmattan, pp. 17-34.

Trehel, N., Blanchet, P. (2003). Pratiques linguistiques régionales et représentations chez des élèves du primaire et de collège en zones suburbaines de Bretagne gallo. Dans: C. Van Den Avenne (Ed.), Contacts de langues (Modèles, typologies, interventions). Paris : L'Harmattan (Collection Espaces Discursifs), pp. 61-78.

Vauvour-Jouve, N. (2001). Toponymie et analyse spatiale en géographie urbaine. Dans : La toponymie urbaine (Significations et enjeux). Paris : L'Harmattan, pp. 141-150.

Veschambre, V. (2006). Marqueurs et mémoires de squats en Europe. Dans: T. Bulot et V. Veschambre (dirs.), Mots, traces et marques (Dimensions spatiale et linguistique de la mémoire urbaine). Paris: L'Harmattan (Collection Espaces Discursifs), pp. 189-204. 